\title{
Reorganization of Visual Processing in Macular Degeneration
}

\author{
Chris I. Baker, ${ }^{1}$ Eli Peli, ${ }^{2}$ Nicholas Knouf, ${ }^{1}$ and Nancy G. Kanwisher ${ }^{1,3}$ \\ ${ }^{1}$ McGovern Institute for Brain Research and Department of Brain and Cognitive Sciences, Massachusetts Institute of Technology (MIT), Cambridge, \\ Massachusetts 02139, ${ }^{2}$ The Schepens Eye Research Institute, Harvard Medical School, Boston, Massachusetts 02114, and ${ }^{3}$ Massachusetts General Hospital \\ (MGH)/MIT/Harvard Medical School Athinoula A. Martinos Center for Biomedical Imaging, MGH, Charlestown, Massachusetts 02129
}

\begin{abstract}
Macular degeneration (MD), the leading cause of visual impairment in the developed world, damages the central retina, often obliterating foveal vision and severely disrupting everyday tasks such as reading, driving, and face recognition. In such cases, the macular damage eliminates the normal retinal input to a large region of visual cortex, comprising tens of square centimeters of surface area in each hemisphere, which is normally responsive only to foveal stimuli. Using functional magnetic resonance imaging, we asked whether this deprived cortex simply becomes inactive in subjects with MD, or whether it takes on new functional properties. In two adult MD subjects with extensive bilateral central retinal lesions, we found that parts of visual cortex (including primary visual cortex) that normally respond only to central visual stimuli are strongly activated by peripheral stimuli. Such activation was not observed (1) with visual stimuli presented to the position of the former fovea and (2) in control subjects with visual stimuli presented to corresponding parts of peripheral retina. These results demonstrate large-scale reorganization of visual processing in MD and will likely prove important in any effort to develop new strategies for rehabilitation of MD subjects.
\end{abstract}

Key words: fMRI; retinotopy; visual cortex; macular degeneration; human; plasticity

\section{Introduction}

In normally sighted subjects, the first cortical areas in the visual pathway (V1-V4) are retinotopically organized, such that adjacent regions of cortex respond to adjacent locations in the visual field (Horton and Hoyt, 1991; Sereno et al., 1995; Engel et al., 1997). In the resulting cortical maps, a large region near the occipital pole, comprising $\sim 20 \mathrm{~cm}^{2}$ of surface area in each hemisphere (the "foveal confluence"), is allocated to just the central $2^{\circ}$ (radius) of visual space (Dougherty et al., 2003). In many subjects with bilateral macular degeneration (MD), the foveal confluence and adjacent cortex is deprived of its normal input as a result of damage to the central retina. We asked whether this deprived cortex simply becomes inactive in subjects with $\mathrm{MD}$, or whether it takes on new functional properties.

Although some reorganization of primary visual cortex has been reported in cats and monkeys after small lesions to peripheral retina (Gilbert, 1998; Kaas, 2002) (but see Horton and Hocking, 1998; Smirnakis et al., 2004), it is not clear that a comparable effect will occur in humans with MD, in which the retinal lesions

Received Aug. 23, 2004; revised Dec. 4, 2004; accepted Dec. 6, 2004.

This work was supported by the Dana Foundation, National Eye Institute Grants EY13455 (N.G.K.) and EY12890 (E.P.), National Science Foundation Catalyst Grant SBE-0350356, and the National Center for Research Resources [P41RR14075, R01 RR16594-01A1 and the National Center for Research Resources Biomedical Informatics Research Network (BIRN) Morphometric Project BIRN002]. We thank J. Haushofer, S. He, M. Mangini, K. Nakayama, H. Op de Beeck, R. Saxe, and G. Yovel for discussion and comments, K. Kwong and T. Benner for technical advice, and F. J. Van de Velde for SLO perimetry.

Correspondence should be addressed to Dr. C. I. Baker, McGovern Institute for Brain Research, Massachusetts Institute of Technology, NE20-443, 77 Massachusetts Avenue, Cambridge, MA 02139. E-mail: cibaker@mit.edu. D01:10.1523/JNEUROSCI.3476-04.2005

Copyright $\odot 2005$ Society for Neuroscience $\quad$ 0270-6474/05/250614-05\$15.00/0 are central and may encompass an area of $>20^{\circ}$ in diameter. In the only study to investigate central retinal lesions (Heinen and Skavenski, 1991), the observed reorganization was weaker than in studies with peripheral lesions. Furthermore, given the extent of the retinal damage common in MD and the large cortical magnification factor for human foveal retina (Sereno et al., 1995), a much bigger area of cortex is affected than in any of these previous studies; any cortical reorganization in humans would have to operate over several centimeters of cortex. The one previous study that investigated retinotopic organization in a single human MD subject (Sunness et al., 2004) reported that cortical areas corresponding to the pericentral scotoma location were silent.

Here, we performed functional magnetic resonance imaging ( $\mathrm{PMRI}$ ) in two MD subjects (MD1 and MD2) with extensive bilateral central retinal lesions and found robust activation in the foveal confluence for visual stimuli falling on peripheral retina. These results demonstrate large-scale reorganization of visual processing in humans with MD.

\section{Materials and Methods}

Subjects. MD1 is a 56-year-old male with early-onset MD (cone-rod dystrophy). Visual acuity was 20/330 in each eye. Macular degeneration was diagnosed in his late 30s. MD2 is a 50-year-old male with an atypical form of juvenile MD. Visual acuity was 20/350 in the better (left) eye. Macular degeneration was diagnosed at age 11.

Visual field plotting. Measurements were conducted in a dimly lit room (0.26 lux; illuminance meter TL-1; Minolta, Osaka, Japan) with screen luminance of $0.021 \mathrm{~cd} / \mathrm{m}^{2}$ and target luminance of $7.0 \mathrm{~cd} / \mathrm{m}^{2}$ (Minolta LS-110 spot photometer). Subjects were seated at an Autoplot Perimeter (Bausch and Lomb, Rochester, NY) facing a white screen $1 \mathrm{~m}$ away. Each 
eye was tested separately. Subjects were instructed to maintain fixation on a fixation point at the center of the screen while a $6 \mathrm{~mm}$ white light target was moved across the screen. People with MD typically adopt a new retinal locus for fixation ["preferred retinal locus" (PRL)] (Timberlake et al., 1986), and subjects fixated with their PRL. Subjects were asked to report whenever the target disappeared. When any scotomatous areas were found, the target was placed inside the scotoma and moved from nonseeing to seeing regions. The point of first seeing the target as reported by the subjects was marked as the edge of the scotoma (supplementary Fig. 1, available at www.jneurosci.org as supplemental material).

Retinal imaging and perimetry. Monocular visual fields, PRL retinal position, and fixation stability were further verified using a Rodenstock (Ottobrunn, Germany) scanning laser ophthalmoscope (SLO) that has integrated microperimetry. First, dynamic perimetry similar to that conducted with the Autoplot was performed but with the advantage of a highly magnified monitoring image of the retina, permitting rejection of any trials in which the subject did not maintain fixation (supplementary Fig. $2 a$, available at www.jneurosci.org as supplemental material). In addition, a static (seen/unseen) procedure was performed in which the retinal location of the fixation target and the stimuli was corrected using a retinal landmark (supplementary Fig. $2 b$, available at www.jneurosci.org as supplemental material).

Functional imaging. Subjects were scanned on a 3.0 T Siemens (Erlangen, Germany) Trio scanner at the Martinos Center for Biomedical Imaging in Charlestown, MA. Functional images were acquired with a Siemens eight-channel phased-array head coil and gradient echo single-shot echo planar imaging sequence $(18-20$ slices; $1.4 \times 1.4 \times 2 \mathrm{~mm}$; interslice gap, $0.4 \mathrm{~mm}$; repetition time, $3 \mathrm{~s}$; echo time, $46 \mathrm{~ms}$ ). Slices were oriented approximately perpendicular to the calcarine sulcus. High-resolution anatomical images were also acquired for each subject for reconstruction of the cortical surface.

Data analysis was performed using Freesurfer and FS-FAST software (http://surfer.nmr.mgh.harvard.edu/). Before statistical analysis, images were motion corrected (Cox and Jesmanowicz, 1999) and smoothed (3 $\mathrm{mm}$ full width at half maximum Gaussian kernel). Activations (stimulus conditions greater than baseline) were visualized on the inflated and flattened cortical surface (Dale et al., 1999; Fischl et al., 1999).

Fixation in MD subjects is typically less accurate than for subjects with normal vision fixating foveally. Therefore, to investigate the organization of visual processing in $\mathrm{MD}$, we devised experiments that, unlike typical retinotopic mapping, do not require extremely precise fixation.

In the first experiment, $\mathrm{MD}$ and control subjects completed four to six runs of a simple blocked-design experiment. Subjects viewed 15-s-long blocks, during each of which images of one category (faces, objects, scenes, or scrambled objects) were presented every $750 \mathrm{~ms}$. Natural images were chosen to approximate everyday vision and to increase the subjects' attention to the stimuli. Each run contained 21 blocks: four for each category and five interleaved baseline periods of no stimuli. Subjects performed a one-back task, responding via a button box any time they saw a consecutive repetition of the same stimulus. Stimuli subtended $\sim 16 \times 16^{\circ}$ of visual angle. MD subjects were instructed to fixate at the center of the visual display with their PRL and maintain steady gaze throughout the experiment. Each control subject was matched to an individual MD subject and was asked to maintain fixation on a point away from the visual display so that stimuli fell on a part of the retina corresponding to the location of the PRL in the matched MD subject. For example, the control subject for MD1 was given a fixation point $10^{\circ}$ above the top edge of the visual display so that the stimuli landed on peripheral retinal positions starting $10^{\circ}$ from the fovea. In MD1, the locations of the scotomata were similar in the two eyes, and this subject and the matched control were tested with both eyes open. However, MD2 and the matched control were tested with the left eye (MD2's better eye) only; the right eye was occluded.

In the second experiment, we compared the cortical response to stimuli presented to the PRL (to replicate the activation of foveal cortex found in the first experiment) and the fovea (to test for any residual foveal function). Both MD1 and MD2, and four control subjects for each MD subject, completed five runs of a blocked-design experiment (21 blocks of $15 \mathrm{~s}$ in each run) in which short visual words (MD1) or objects (MD2)
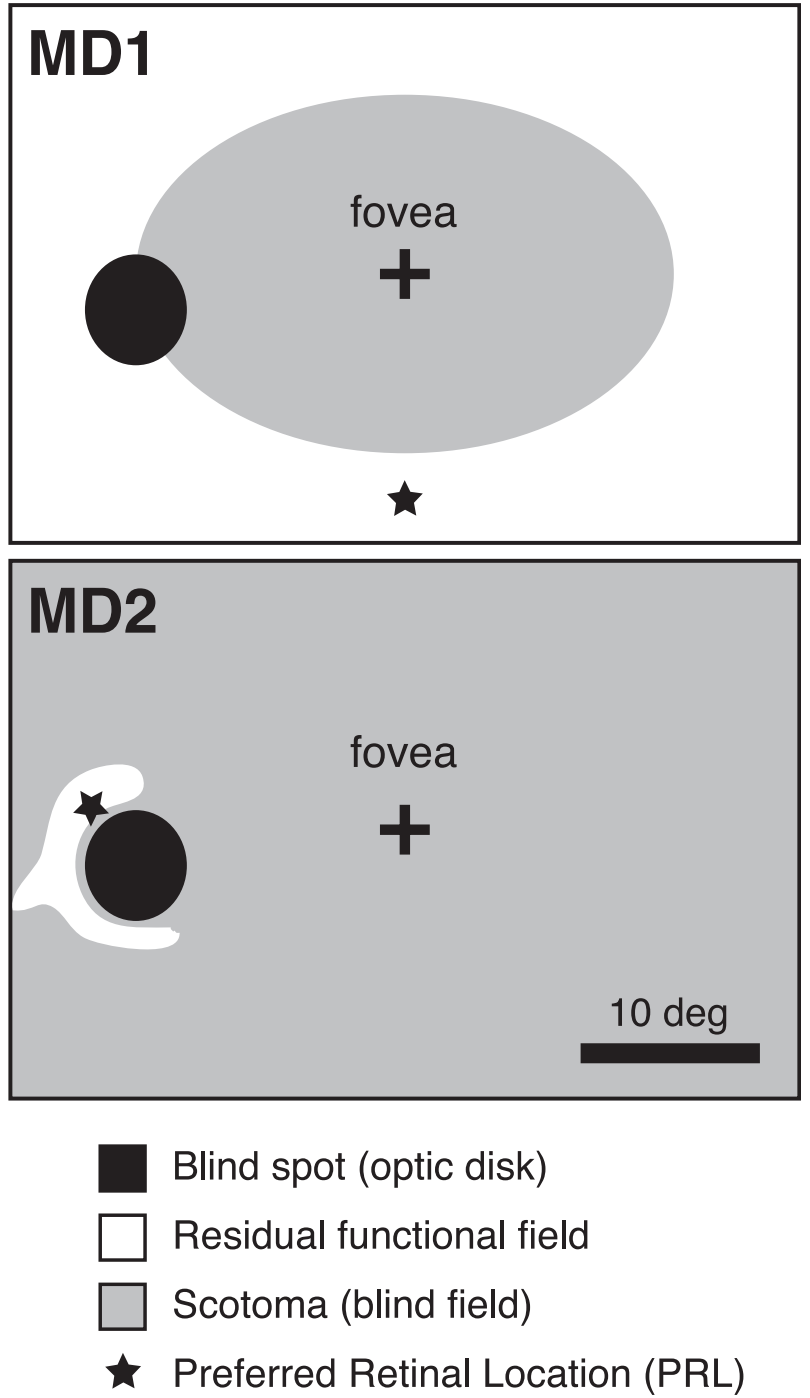

Figure 1. Schematic diagram indicating visual fields in the left eyes of MD1 and MD2. Only the central $44 \times 30^{\circ}(\mathrm{deg})$ is shown. In MD1, all retina outside this region is functional; in MD2, some functional retina exists outside this region.

were presented either to the fovea (foveal location) or to an eccentric retinal location corresponding to the PRL (PRL location), with additional periods of no stimulus presentation to serve as a baseline. MD1 and control subjects viewed the stimuli passively, whereas MD2 and controls performed a one-back task. All subjects viewed stimuli with one eye only (MD1 and controls, right eye; MD2 and controls, left eye); the other eye was patched. Words subtended $12 \times 5^{\circ}$ of visual angle on average, and objects subtended $8 \times 10^{\circ}$ on average. The edges of the stimuli at the two locations were separated by $8^{\circ}$ vertically in MD 1 and by $9.5^{\circ}$ horizontally in MD2. For MD subjects, a fixation point $\left(\sim 1.7^{\circ}\right.$ in diameter) was presented at the PRL location, and the subject fixated here with their PRL (effectively placing their former fovea at the foveal location). For control subjects, a fixation point $\left(\sim 0.3^{\circ}\right.$ in diameter) was placed at the foveal location, and subjects fixated there with their fovea. This effectively placed a portion of their peripheral retina, corresponding to the location of the respective MD subject's PRL, at the PRL location.

To measure the magnitude of the response in foveal cortex to stimuli presented to peripheral versus foveal locations, a region of interest (ROI) was defined for both hemispheres of all subjects based on anatomical criteria. ROIs were drawn at the posterior end of the calcarine sulcus, and the surface area of the ROI in each hemisphere was $\sim 200 \mathrm{~mm}^{2}$ (range, 191-213 $\mathrm{mm}^{2}$ ), $\sim 1 / 10$ the total surface area of the foveal confluence.

To verify the ability to fixate stably during this second experiment, eye 
movements were monitored in MD1 outside of the scanner during two runs identical to those he saw in the scanner. Eye movements were monitored with an ISCAN (Burlington, MA) model RK726PCI pupil/corneal reflection tracking system equipped with an RK620-PC autocalibration system. The ISCAN device has a nominal accuracy of $0.3^{\circ}$ over a $\pm 20^{\circ}$ range and a sampling rate of $60 \mathrm{~Hz}$ and was calibrated using a five-point calibration scheme.

\section{Results}

\section{Retinal data}

In both subjects, foveal vision had been completely eliminated in both eyes by MD (Fig. 1). Central vision loss in MD1 extended $>10^{\circ}$ from the former fovea in both eyes. In MD2, in the better (left) eye, the nearest functional retina to the former fovea was over $17^{\circ}$ away. In MD1, the PRL was below the central scotoma (12.5 and $13.5^{\circ}$ from the fovea in the left and right eye, respectively). In MD2, in the better eye, this was $17.5^{\circ}$ lateral to the fovea, close to the optic disk (on the nasal retinal side).

\section{fMRI data}

In the first experiment, for both subjects (Fig. 2), visual stimulation, compared with the blank screen baseline, strongly activated the foveal confluence and adjacent cortical regions corresponding to the projection zone of the damaged retina (see supplementary Fig. 3, available at www.jneurosci.org as supplemental material, for a schematic diagram showing the organization of visual cortex on the flattened cortical representation). Because MD2 was tested with the right eye patched, and his PRL is in nasal retina, stimuli mainly landed in his left visual field, and data are shown for his right hemisphere only. The only prominent activations in the left hemisphere were in nonretinotopic, high-level visual cortex (see also supplementary Fig. 4, available at www.neurosci.org as supplemental material).

Specifically, in both subjects, robust activation was observed at the posterior end of the calcarine sulcus extending laterally and ventrally (Fig. $2 a, d$ ). These activations were present even with conservative statistical thresholds (e.g., $p<10^{-10}$ ). Because MD has eliminated foveal vision in these subjects, the activation in cortical regions normally responsive only to foveal input must result from visual stimulation of peripheral retina.

Although it is difficult to distinguish V1, V2, V3, and V4 within the foveal confluence (Dougherty et al., 2003), the observed activations extended into the depths of the calcarine sulcus and clearly include V1 (Stensaas et al., 1974; Rademacher et al., 1993; Andrews et al., 1997) as well as adjacent retinotopic regions. Activation was also observed in the region of visual cortex that normally responds to stimuli falling on peripheral retina (corresponding to the location of each subject's PRL) (Fig. 2, arrows), and in MD2, this activation appears to be spatially separated from activation at the foveal confluence. In addition, ventral activations extended anteriorly onto inferior temporal cortex, reflecting activation of object-selective regions.

\section{MD1}

MD2 sulcus

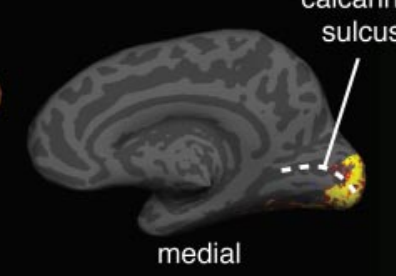

d

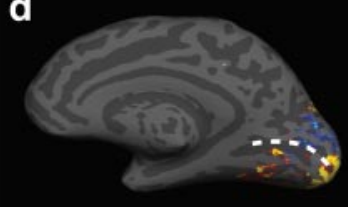

e

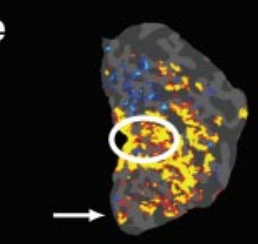

foveal
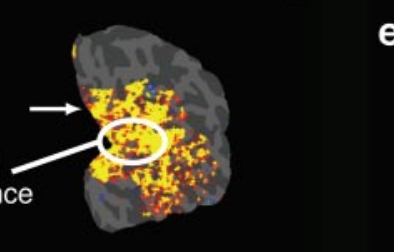

$\longrightarrow$

f Control

Control

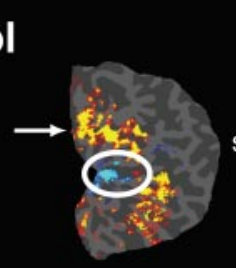

$\mathbf{f}$

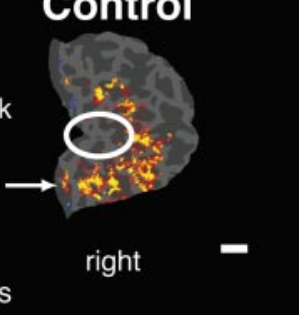

Figure 2. Activation of visual cortex (stimuli greater than baseline) in MD and control subjects (experiment 1). $a$, Ventral and medial views of inflated right hemisphere of MD1. $b$, Flattened occipital cortex of MD1 showing activation at the foveal confluence. (a) confluence. $f$, Flattened occipital cortex of a control subject matched to MD2. Note the absence of activation in the foveal confluence for stimuli presented to peripheral retina (equivalent to MD2's PRL). Arrows point to activations overlying cortex that would normally be responsive to peripheral stimuli presented at retinal locations corresponding to the PRLs. Scale bar, $20 \mathrm{~mm}$.

The activation of the foveal confluence and adjacent cortex by peripheral stimuli was not observed in matched control subjects. In neither control subject was any significant activation observed near the occipital pole for peripheral stimulus presentation compared with baseline (Fig. $2 c, f$ ). In fact, activation in the region of the foveal confluence was lower during periods of stimulus presentation than during the baseline periods for the control subject for MD1 (Fig. $2 c$, blue regions), consistent with previous reports of negative blood oxygenation level-dependent responses in foveal cortex with peripheral attention (Somers et al., 1999).

In the second experiment, we tested the possibility that the activations we observed in the first experiment were attributable to residual foveal function by presenting stimuli either just to the PRL or just to the fovea. If typically foveal cortex is responsive to peripheral visual stimuli, we would expect to see activation of the foveal confluence for visual presentation to the PRL but no activation for presentation to the fovea. This is exactly what we observed in both MD subjects. Whereas stimuli presented to each MD subject's PRL (compared with the blank screen baseline) strongly activated the foveal confluence and adjacent cortex, stimuli presented to the former fovea did not elicit any significant activity in visual cortex (Fig. 3a). This finding rules out the possibility that residual foveal function contributed to the activations observed in the first experiment. In MD1, eye movement monitoring outside of the scanner confirmed that he was able to 


\section{a Stimuli at PRL Stimuli at fovea b}
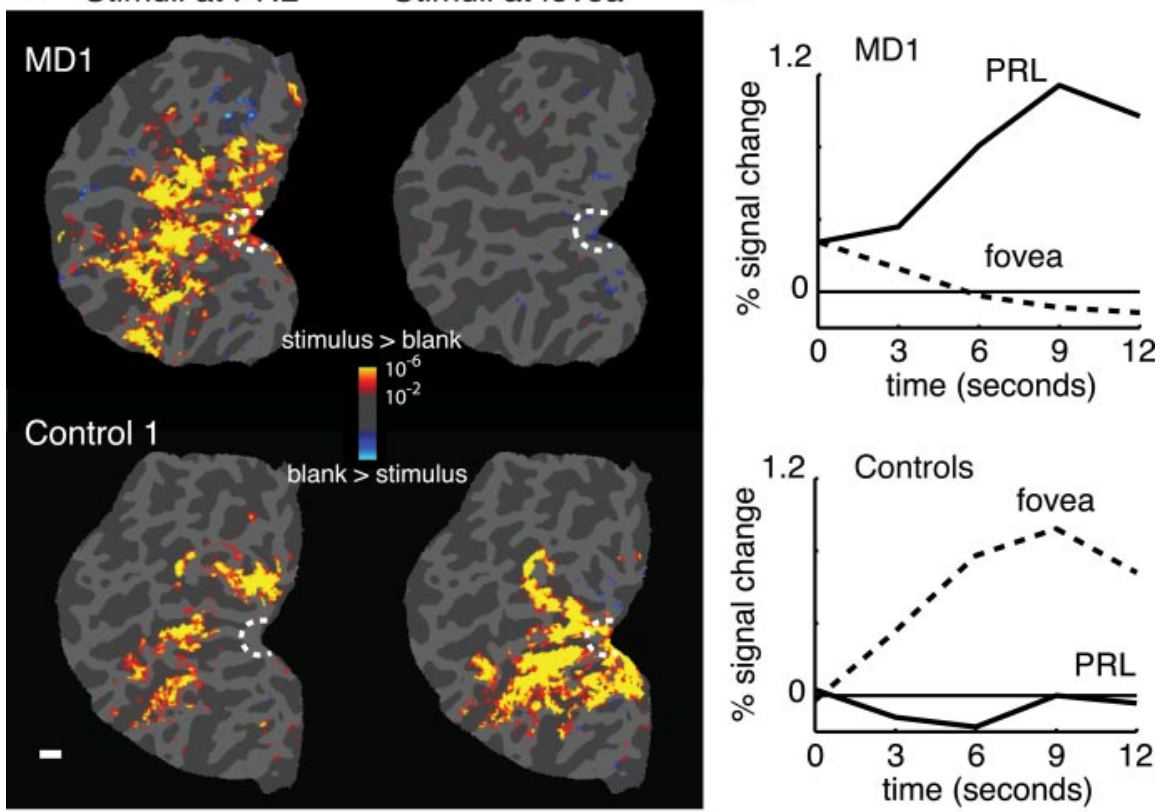

C

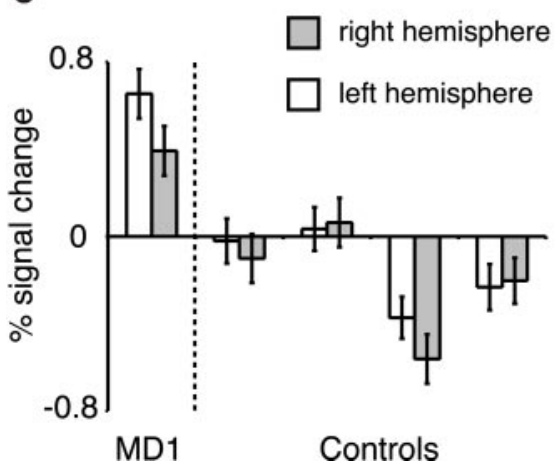

d

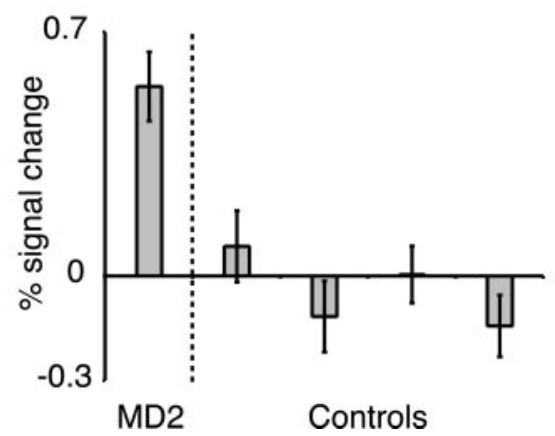

Figure 3. Visual activation in MD and control subjects to stimuli presented either at the fovea or at the PRL (experiment 2). a, Left hemisphere of MD1 and one control subject. Peripheral, but not foveal, stimuli elicited strong activation in foveal cortex in MD1. In contrast, foveal cortical activation was only observed with foveal presentation in the control subject. Scale bar, $10 \mathrm{~mm}$. $b$, Time course of response in an anatomically defined ROI at the posterior end of the calcarine sulcus (dashed white outlines in $a$ ) in MD1 (top) and four control subjects (bottom). $c, d$, Average responses to peripheral stimuli in foveal cortical ROIs in MD and control subjects. For MD1 and controls, stimuli were presented at the midline, and data are shown from both hemispheres. For MD2 and controls, stimuli mainly landed in the left visual field, and data are shown for the right hemisphere only. Error bars indicate SE.

maintain stable fixation for the duration of the experimental runs. Throughout the two runs, the maximum vertical deviation of the eye from the center of the fixation point was $3.4^{\circ}$, whereas the distance between the edges of the stimuli at the foveal and PRL locations was $8^{\circ}$. In MD2, fixation stability measurements collected with the SLO showed that the typical variation in fixation position is much less than the $10^{\circ}$ that separated the edges of the stimuli at the foveal and PRL locations. Note that unstable fixation could not result in activation of foveal cortex, because there was no foveal cortical activation even under direct stimulation of the fovea.

In the control subjects for each MD subject, as expected, stimuli presented at the fovea elicited strong activity in the foveal confluence and adjacent cortex, but stimuli presented at the PRL location elicited no significant positive activation in this region (Fig. 3). In a small ROI defined at the occipital pole in all subjects, only the MD subjects showed significantly increased activation, compared with the baseline condition, for visual stimuli pre- sented to peripheral retina (Fig. $3 c, d$ ). Thus, peripheral and not foveal visual stimuli elicit activation of the foveal confluence in MD subjects, whereas no significant positive visual activation is elicited in the foveal confluence with equivalent peripheral stimuli in control subjects.

\section{Discussion}

Our results demonstrate large-scale reorganization of visual processing in humans after large, central retinal lesions. Strong activation of normally foveal cortex by peripheral visual stimuli was observed in $\mathrm{MD}$ subjects, despite the facts that (1) foveal visual stimuli produce no cortical activations at all in these subjects, and (2) peripheral stimuli produce no activation of foveal cortex in normal subjects. This reorganization was demonstrated in subjects that had lost their foveal vision late in adulthood (MD1) or late in childhood (MD2).

These findings critically extend previous results obtained in cats and monkeys showing some reorganization in V1 after peripheral retinal lesions (Gilbert, 1998; Kaas, 2002). Not only have we demonstrated reorganization of visual processing in humans after retinal lesions, but we have shown that reorganization can occur even when tens of square centimeters of surface area of cortex are affected, much more than in the previous nonhuman studies. The failure to find evidence for reorganization in a previous study of a single subject with MD (Sunness et al., 2004) may be a result of the sparing of the fovea in that subject or to the far shorter time since the onset of MD (3 years) compared with MD1 and MD2 (>20 years). However, it is worth noting that the conclusion in this previous study is based on data from one hemisphere in a single $8 \mathrm{~min}$ imaging run. Furthermore, although the authors report a region of cortex with no visual activation, there is nonetheless some activation in cortical locations corresponding to the scotomata. Although previous studies (Morland et al., 2001; Baseler et al., 2002) have reported activation of foveal cortex in humans with congenital rod monochromacy, vision loss is difficult to assess reliably in such subjects, is confined to a very small area at the center of the fovea $\left(<1^{\circ}\right)$, and any changes in visual processing in these subjects could reflect early developmental plasticity.

Three possible mechanisms could account for the activations we observed in foveal cortex by peripheral stimuli. First, studies of cortical reorganization in V1 after peripheral retinal lesions in cats and monkeys have identified intrinsic horizontal connections as a likely substrate (Darian-Smith and Gilbert, 1994; Das and Gilbert, 1995), spreading activation from areas receiving sensory input to cortical areas deprived of input. However, because of the high cortical magnification factor in human foveal visual cortex and the large size of the retinal lesions in our subjects, to 
account for our data, horizontal connections would have to spread activation across several centimeters of cortex, farther than the length of typical horizontal connections in primate V1 (6-8 mm) (Gilbert et al., 1996; Angelucci et al., 2002); a polysynaptic chain of horizontal connections would be required. Second, reorganization could result from changes at an earlier level of the visual system than $\mathrm{V} 1$, such as the lateral geniculate nucleus (LGN), modifying input into V1. This seems unlikely, however, given that studies of cats and monkeys with retinal lesions have found minimal reorganization in the LGN (Eysel et al., 1980; Gilbert and Wiesel, 1992) (but see Florence et al., 2000). A third potential locus for reorganization is top-down feedback from higher-level areas. We found no evidence for such top-down feedback into foveal cortex in normal subjects in our experiments, but top-down influences on retinotopic cortex have been reported in work on attention (Somers et al., 1999) and mental imagery (Klein et al., 2000), and these effects could be strengthened in people with MD. Furthermore, many studies have reported activation of visual cortex in nonvisual tasks in blind subjects (Burton et al., 2002a,b; Amedi et al., 2003), presumably reflecting changes in top-down input to visual cortex, although this phenomenon may be restricted primarily to cases of earlyonset blindness (Sadato et al., 2002) and could therefore reflect early developmental plasticity. Additional studies will be needed to determine the mechanism of reorganization of visual processing in MD.

In summary, we have demonstrated activation of foveal cortex by peripheral visual stimuli in subjects with MD. This foveal activation is centimeters away from the region of cortex that normally responds to peripheral stimuli, indicating a large-scale reorganization of visual processing in human adults deprived of foveal vision. Future research will test the consequences of this reorganization for visual performance (Nugent et al., 2003) as well as testing the mechanisms underlying the reorganization by investigating the time course of the phenomenon. Whatever the answers to these questions, the fact that visual cortex can take on new functions and can remain responsive decades after its normal retinal input has been removed will undoubtedly prove important in any effort to develop new strategies for rehabilitation of MD subjects.

\section{References}

Amedi A, Raz N, Pianka P, Malach R, Zohary E (2003) Early "visual" cortex activation correlates with superior verbal memory performance in the blind. Nat Neurosci 6:758-766.

Andrews TJ, Halpern SD, Purves D (1997) Correlated size variations in human visual cortex, lateral geniculate nucleus, and optic tract. J Neurosci 17:2859-2868.

Angelucci A, Levitt JB, Walton EJ, Hupe JM, Bullier J, Lund JS (2002) Circuits for local and global signal integration in primary visual cortex. J Neurosci 22:8633-8646.

Baseler HA, Brewer AA, Sharpe LT, Morland AB, Jagle H, Wandell BA (2002) Reorganization of human cortical maps caused by inherited photoreceptor abnormalities. Nat Neurosci 5:364-370.

Burton H, Snyder AZ, Conturo TE, Akbudak E, Ollinger JM, Raichle ME (2002a) Adaptive changes in early and late blind: a fMRI study of Braille reading. J Neurophysiol 87:589-607.

Burton H, Snyder AZ, Diamond JB, Raichle ME (2002b) Adaptive changes in early and late blind: a FMRI study of verb generation to heard nouns. J Neurophysiol 88:3359-3371.

Cox R, Jesmanowicz A (1999) Real-time 3D image registration for functional MRI. Magn Reson Med 42:1014-1018.

Dale AM, Fischl B, Sereno MI (1999) Cortical surface-based analysis. I. Segmentation and surface reconstruction. NeuroImage 9:179-194.
Darian-Smith C, Gilbert CD (1994) Axonal sprouting accompanies functional reorganization in adult cat striate cortex. Nature 368:737-740.

Das A, Gilbert CD (1995) Long-range horizontal connections and their role in cortical reorganization revealed by optical recording of cat primary visual cortex. Nature 375:780-784.

Dougherty RF, Koch VM, Brewer AA, Fischer B, Modersitzki J, Wandell BA (2003) Visual field representations and locations of visual areas V1/2/3 in human visual cortex. J Vis 3:586-598.

Engel SA, Glover GH, Wandell BA (1997) Retinotopic organization in human visual cortex and the spatial precision of functional MRI. Cereb Cortex 7:181-192.

Eysel UT, Gonzalez-Aguilar F, Mayer U (1980) A functional sign of reorganization in the visual system of adult cats: lateral geniculate neurons with displaced receptive fields after lesions of the nasal retina. Brain Res 181:285-300.

Fischl B, Sereno M, Dale A (1999) Cortical surface-based analysis. II: Inflation, flattening, and a surface-based coordinate system. NeuroImage 9:195-207.

Florence SL, Hackett TA, Strata F (2000) Thalamic and cortical contributions to neural plasticity after limb amputation. J Neurophysiol 83:3154-3159.

Gilbert CD (1998) Adult cortical dynamics. Physiol Rev 78:467-485.

Gilbert CD, Wiesel TN (1992) Receptive field dynamics in adult primary visual cortex. Nature 356:150-152.

Gilbert CD, Das A, Ito M, Kapadia M, Westheimer G (1996) Spatial integration and cortical dynamics. Proc Natl Acad Sci USA 93:615-622.

Heinen SJ, Skavenski AA (1991) Recovery of visual responses in foveal V1 neurons following bilateral foveal lesions in adult monkey. Exp Brain Res 83:670-674.

Horton JC, Hocking DR (1998) Monocular core zones and binocular border strips in primate striate cortex revealed by the contrasting effects of enucleation, eyelid suture, and retinal laser lesions on cytochrome oxidase activity. J Neurosci 18:5433-5455.

Horton JC, Hoyt WF (1991) The representation of the visual field in human striate cortex. A revision of the classic Holmes map. Arch Ophthalmol 109:816-824

Kaas JH (2002) Sensory loss and cortical reorganization in mature primates. Prog Brain Res 138:167-176.

Klein I, Paradis AL, Poline JB, Kosslyn SM, Le Bihan D (2000) Transient activity in the human calcarine cortex during visual-mental imagery: an event-related fMRI study. J Cogn Neurosci 12 [Suppl 2]:15-23.

Morland AB, Baseler HA, Hoffmann MB, Sharpe LT, Wandell BA (2001) Abnormal retinotopic representations in human visual cortex revealed by fMRI. Acta Psychol (Amst) 107:229-247.

Nugent AK, Keswani RN, Woods RL, Peli E (2003) Contour integration in peripheral vision reduces gradually with eccentricity. Vision Res 43:2427-2437.

Rademacher J, Caviness Jr VS, Steinmetz H, Galaburda AM (1993) Topographical variation of the human primary cortices: implications for neuroimaging, brain mapping, and neurobiology. Cereb Cortex 3:313-329.

Sadato N, Okada T, Honda M, Yonekura Y (2002) Critical period for crossmodal plasticity in blind humans: a functional MRI study. NeuroImage 16:389-400.

Sereno MI, Dale AM, Reppas JB, Kwong KK, Belliveau JW, Brady TJ, Rosen BR, Tootell RB (1995) Borders of multiple visual areas in humans revealed by functional magnetic resonance imaging. Science 268:889-893.

Smirnakis SM, Brewer AA, Schmid M, Tolias AS, Augath M, Inhoffen W, Shüz A, Wandell BA, Logothetis NK (2004) V1 cortical reorganization revisited: fMRI and electrophysiology in macaque following retinal lesions. Soc Neurosci Abstr 30:605.3.

Somers DC, Dale AM, Seiffert AE, Tootell RB (1999) Functional MRI reveals spatially specific attentional modulation in human primary visual cortex. Proc Natl Acad Sci USA 96:1663-1668.

Stensaas SS, Eddington DK, Dobelle WH (1974) The topography and variability of the primary visual cortex in man. J Neurosurg 40:747-755.

Sunness JS, Liu T, Yantis S (2004) Retinotopic mapping of the visual cortex using functional magnetic resonance imaging in a patient with central scotomas from atrophic macular degeneration. Ophthalmology 111:1595-1598.

Timberlake GT, Mainster MA, Peli E, Augliere RA, Essock EA, Arend LE (1986) Reading with a macular scotoma. I. Retinal location of scotoma and fixation area. Invest Ophthalmol Vis Sci 27:1137-1147. 